\title{
A Turn in the Road of Media Studies
}

\author{
William Uricchio
}

Massachusetts Institute of Technology, USA

\begin{abstract}
Whether in the form of Google searches, interactive games, or responsive textual environments, the reassuring subject-object binary so fundamental to the modern era's representation systems is fast slipping away. In its place, a recursive epistemological order that actively parses the subject and shapes the textual world is fast emerging, posing challenges to established notions of agency and to narrative as a cultural operating system. Assessments of the terms and implications of this shift will benefit from the distinctive analytic perspective that distinguishes the Nordic from many of its Anglo-American and European peers. Keywords: narrative, algorithmic, agency, interactive, recursive
\end{abstract}

\section{Introduction}

Before proceeding with a few comments regarding directions that the Nordic Journal of Media Studies might take in the foreseeable future, I'd like to offer a proviso regarding the modifier "Nordic". "Nordic" marks a cultural space of which I am not a part, though it is one I have been involved in through personal friendships and as a partner in various intellectual endeavours over the years, and one that I have benefitted from enormously. It's a cultural assertion of some complexity, as I learned from time spent lecturing and in visiting professorships in the nations that constitute the Nordic. It looks and feels quite distinctive when viewed from within any one culture, and is probably at its most coherent and stable, if superficial, when viewed from the outside. And it's a modifier of great flexibility in the context of the journal, since it can be construed variously as a claim regarding region, perspective, domain, or ethos.

What does stand out to me is "Nordic" as an approach to media that productively intersects domains called, in the American context from which I write, media studies (firmly embedded in the humanities) and communication studies (ensconced in the social sciences). These are awkward distinctions, of course, playing out differently in various national academic traditions, where even the

Uricchio, W. (2020). A turn in the road of media studies. Nordic Journal of Media Studies, 2, 157-166. https://www.doi.org/10.2478/njms-2020-0014 
same terms can mean quite different things. The work of scholars such as Kim Schrøder, Birgitta Høijer, Kirsten Drotner, Jostein Gripsrud, Johan Fornäs, and Klaus Bruhn Jensen, among many others, has brilliantly triangulated the work of American, British, and Continental (particularly Germanic) media and communications scholars, rising above them to offer incisive interventions for the framing of the field, parsing them through distinctive intellectual traditions, and generating contributions, both empirical and theoretical, to the understanding of media's operations.

While the situation I will discuss is largely global in its operations, and while it will be approached in predictable ways by the dominant national "schools", I very much look forward to what will emerge from the work within the Nordic tradition. As has already happened with television studies, that work will be generative for those of us inhabiting other thought systems and national academies. To be clear, rather than a place or set of regional dynamics, I wish in this article to invoke the Nordic as a set of analytic perspectives.

With this Nordic "stance" in mind, I'll turn to a few developments I think will increasingly demand attention in the coming years - but not before mentioning a second proviso: Media specialists in the early twenty-first century - like the larger human species - face an extraordinary number of interrelated challenges in which media will play a central role. These include climate change; economic and political polarisation; globalised ownership, labour, peoples, and cultures; and the decline of long-established governance structures (religion, civic society, and the nation-state). Rather than taking on these deserving topics, I'd like to focus my comments on what I see as a fundamental and even epistemic challenge to media and the social relationships bound up in media enactments and explore a few of the ensuing implications, including a reconfiguration of the subject's position, the recursive production of audiences and texts, the changing condition of narratives, and in short, what might be termed an epistemic crisis in media. This new situation, I will argue, recasts the knowledge accumulated over the years in media studies, and marks the space where the Nordic approach to the field can make an important intervention. This is admittedly the tip of the iceberg, but one has to start somewhere!

\section{The subject's position}

The history of modern media in the commonplace sense might be located in Mainz, with Guttenberg's mid-fifteenth century printing press. ${ }^{1}$ It roughly coincided with the formalisation of three-point perspective, and both of these developments aligned with the formation of the modern subject in terms that Heidegger would go on to describe in his essay on the Weltbild [world picture]. A representational order took hold in the West that is reified in the modern era's media technologies and modes of deployment. And that order is predicated on a clearly defined binary of subject and world. 
As I've argued in various essays over the past few years, this is now changing (e.g., Uricchio, 2011). To say it boldly, the contours of an epistemic shift, the likes of which Western culture has not experienced for five and a half centuries, are emerging. This is a big claim, of course, and I make it fully aware of the recurrence of apocalyptic cries heralding the appearance of each new media form: the printing press, photography, telegraphy, telephony, film, and the broadcasting media each provoked fears of radical change. But claims or not, all shared a pattern of deployment that essentially served to amplify the producing subject. All worked to maintain the coherence of the binary relationship that Heidegger saw as defining the (long) modern.

For the first time in a half millennium, something different is happening. The reassuring binary so fundamental to the modern era's representation systems is steadily giving way to a new epistemological order that is recursive in nature, that actively parses the subject and shapes access to the world in ways that are neither evident nor perhaps even knowable. Now as with the dawn of the modern era, the shift is bound up in deployments of technology - in today's case, the algorithm, which I mean in the broad sense to include the constellation of data, modelling, training sets, and the rest of the operations that undergird much of so-called new media. (I use this term as a synecdoche, echoing analysis of the term's use by Tarleton Gillespie, 2016.) Whether curating search results, book recommendations, or music selections; enabling social media platforms and shaping news feeds; recognising faces, behaviours, and potentially acting upon those determinations; constructing immersive and responsive worlds in virtual reality; and more, an algorithmic regime not only stands between the subject and the world apprehended, but actively shapes access to the world in recursive conversation with the subject and whatever biases the system carries by design. This is a radical new condition, and ruptures precisely the subject-object relationship that defines the modern and is embedded in five hundred plus years of media use. To be clear, what's "radically new" about today's social media networks, for example, is not the network, which has precedents in telegraphy and telephony; nor is it the blurring of the familiar binary of producer and consumer, which has occurred historically across media forms in various co-creation configurations (for a closer look at co-creative practices, see Cizek \& Uricchio, 2019). What is new and disruptive of the modern's defining subject-object relationship is the recursive character of these new media forms. Rather than simply extending, connecting, or multiplying the textual utterances of the subject, this emergent class of media is responsive, learning the subject's preferences and shaping the textual world to which the subject has access according to the system's optimisation algorithms. Users don't simply “see” one another's feeds on social media; rather, they see a constantly evolving curation of those feeds that reflects an assessment of what will best serve the system's operating imperatives together with an ongoing assessment of the subject's responses.

If the "radically new" in this formulation is the repositioning of agency in the guise of the recursive and "personalised", why do media scholars and the press 
focus on networks, the blurring of the production-consumption binary, and the organisational logics behind Facebook and Google? I think it reflects the role of precedent; these are well-trodden categories from earlier media experience. They have a familiar salience, but habituation encourages a cultural stance in which the new can easily be retrofitted into the familiar contours of the old, enabling radical potentials to be occluded. The relations implicit in the modern (and again, Heidegger is as eloquent as efficient in mapping them) have acquired a taken-forgrantedness that infuses knowledge and informs ways of navigating the new, even at the risk of missing what is truly radical.

This broad shift from the old certainties to a new recursive order gives me great sympathy for those who witnessed the social and epistemological upheaval associated with the printing press and three-point perspective in the fifteenth century, the previous "disruption" and the one that emblematises the notion of the modern that I have been invoking and that is now at risk. Adrian Johns's wonderful work on the history of the book shows how destabilising the experience was, and how ill-prepared the cultural frame for those "backing into" the Early Modern from the Medieval Era (Johns, 1998).

What does this mean for the future of media and their embeddedness in daily life? And what does it mean for the field, from teaching and research agendas to the Nordic Journal of Media Studies? For starters, it means rethinking the conceptual frames for assessing recursive and responsive processes of mediation - in other words, today's "new media”. To the extent that the subject's data - whether historical, such as past browsing and past purchases, or behavioural, such as facial gestures and eye movements - inform the algorithms responsible for curating the textual world to which users have access, a new dynamic is at play. No longer gatekeeping, filtering, or distortion in the usual sense, these dynamics are fundamentally personal (although their predictive power emerges from correlating individual data against massive social data sets) and increasingly responsive (using the subject's data to fine-tune system interactions). How should agency be conceptualised in these systems where no particular human is responsible for system behaviours? How to imagine relationality in a mediascape that increasingly responds to and anticipates some notion of the subject? What is the nature and status of the text in a system where textual possibility and context are malleable and potentially individualised? Do concepts like communication accurately capture scenarios in which the sender has no idea which of their posts will really be sent, or when the receiver cannot know with assurance which of their behaviours is being monitored, monetised, and ultimately used to shape the media feed? The relative clarity of the West's inherited "world picture" contrasts sharply with the opacity introduced by these emergent systems.

These fast-changing and recursive relationships raise profound questions about human agency, autonomy, and privacy - will these developments enhance, diminish, or otherwise modify the subject's responsibilities and range of action? Their crafting of an individuated and responsive world challenges existing social 
defaults such as inherited value systems and hierarchies of authority. Will we see continuity or breaks in the social order and notions of justice, human rights, privacy, and authenticity - and with what implications? They pose questions about human singularity: how entangled is the notion of subjectivity with technology, and what is the nature of the subject's relationship with these algorithmic systems? And - perhaps most importantly - what new language will enable these questions to be asked and who will be empowered to interrogate and critically assess the new relationships enabled by this emergent order and its mediations of the world? The last point is perhaps the most important if this new condition is to be assessed critically, rather than being forced back into the categories and logics of the past.

Continuities mask ruptures, perhaps inevitably. Although I am arguing for the need to consider a new situation, I am also aware that inherited linguistic and theoretical frames necessarily impinge upon all that is radical in that newness. It is perhaps a good moment to return to the likes of Thomas Kuhn and others who have analysed the dynamics of paradigm shifts, and charted historical responses.

\section{Audiences, texts, and stories}

Over the years, media studies broadly-writ have cycled through theories of the audience-text relationship, along the way encountering pliable audiences (media effects), active audiences (from uses and gratifications to various interventions of cultural studies), to what might be termed productive audiences (as users navigate through textual environments like games or even contribute textual elements in settings like YouTube). Given what I've just argued about an emerging recursive regime, it is worth speculating about an audience form that, for want of a better term, I'll call algorithmic. Algorithmic audiences, to play this out, take two forms: as individually targeted receivers of texts; and as indirect generators of texts. In the first case, visible in the operations of companies such as Facebook and Cambridge Analytica, particular texts are vetted and directed to particular users based on assessments of the user's profile. One might say that the audience is algorithmically curated on a level approaching the individual. The textual composite the user sees - the mix of advertisements, news articles, postings from friends, and so on - bears little resemblance to what is "transmitted" (if one can even speak in these terms given the overabundance and non-sequential nature of the feed). Rather, past is prologue in a system in which accreted behaviours inform the textual selection process, and responses to that selection are incorporated in real time adjustments by a recursive system that privileges factors unknown to the user. To the extent that the system is responsive, strategic, and adaptive, it might be deemed intelligent. Alas, that intelligence serves another master, even if it exists in a constitutive relationship to its audience.

The second notion of this possible algorithmic audience goes a step further and generates texts-on-demand for the user. It is related in a way to the justmentioned productive audience, particularly as manifest in the navigational work 
of interactive texts (or in the case of my lab at MIT, documentaries ${ }^{2}$ ). But rather than the user deciding where to go and what to view, the system, again using backlogged user data, acts as a third party, standing between the audience and the text, brokering the two by extrapolating insights from user data and using them to construct the text. The audience simply experiences a made-to-measure linear text that generally masks the fact that it has been compiled on-the-fly algorithmically. ${ }^{3}$ Obviously, this scenario is riddled with significant ethical challenges regarding agency and privacy. The agency issues are currently being debated in the domain of self-driving cars; but the privacy issues, in which the user's gaze and bio-markers are datafied, interpreted, and presumably monetised as signs of interest, go far beyond the familiar data trails that are currently the subject of policy debates.

The curation of the audience-text relationship is already manifest in small and insidious ways, whether in the form of Google searches (curated in terms of language, geographical location, and doubtless many other markers), Facebook feeds, music prediction systems (Spotify), and literary and film and television tastes (Amazon, Netflix) (Uricchio 2017). This "personalisation" (to put it in the innocuous terms of the past) continues apace in the domain of more traditionally defined "texts". In the realm of print, companies such as Narrative Science, Yseop, and Automated Insights mine and analyse data, using natural language processing to deliver it to the user (potentially on an individualised basis) as story. Although still primarily deployed in business settings and by the press for structured data sets (sports and finance), these systems are perfectly capable of reporting on sporting events with stories uniquely configured for each participant. Video-based story systems are fast catching up. This technology is developing quickly, as evidenced by the Stanford-Adobe "automated" video editing system, and in ways that require a critical stance, including advances in image and sound synthesis manifest in DeepFake.

Responsive textual systems with their algorithmic mediation of the audience pose a new order of questions across media forms. Even if - to the algorithm the audience appears as both a highly individuated data set and a responsive rule set for textual construction, it nevertheless yields flesh-and-blood audiences and recursively produced real-world texts. It breaks the audience-text (and subjectworld) binary by introducing an intermediary element that determines both - and in so doing, marks a new dynamic.

What might this mean for narrative? Audiences and producers of texts have drawn on narrative as a cultural coding protocol for a very long time, as Vladimir Propp demonstrated. Bibles, Korans, and the other great books suggest a deep history of narrative as bearers of cultural operating systems, encoding norms and values into textual systems that have survived centuries. It's no surprise, then, that media studies have long been interested in narrative; and that interest in turn has benefitted from well-established theories used in literature. Film and television, like the printed word, share a similar condition: the narrative is by default fixed to 
a physical medium, its sequence of events "hard wired" to a platform. Of course, it is possible to subvert that fixity, as demonstrated by examples from Cortazar's Hopscotch, where the reader is free to jump around inside the text, to Warhol's Chelsea Girls, with two unsynchronised screens and a variable soundtrack. But by and large, novels, film, and television programmes all hew to the intertwined stability of platform and narrative. Enter digital games, with rule-bound enactments (play) and genres, but minimal narrative fixity. Players negotiate the rules, navigate ludic space, and (genre depending) generate their own narrative experience. Narrative, that is to say, can be fixed to a particular narrative sequence; but it can also be experientially extrapolated by the user navigating a textual environment. Media studies have largely preferred the former notion of narrative; and communication studies (particularly reception studies) have shown greater interest in the latter. The continued development of interactive digital media, from games to virtual reality, where a given textual environment supports myriad narrative pathways, has amplified the importance of thinking about user-activated narratives.

These scenarios - whether algorithmically generated linear narratives for individual users or environments encouraging multiple narrative encounters - subvert narrative's historical role as a means of sharing the experience of others and as an experience to be shared in common with others. To the extent that societies store and transmit their operating systems in the form of stories across multiple generations and even millennia, what implications will responsive customisation and individualised texts have for the social cohesion of the future?

\section{Epistemological uncertainty}

Despite Donald Trump's tiresome invocations of “fake news" when confronted with unflattering reports, legacy American print and broadcast news operations are generally accurate and empirically grounded, even if ideologically skewed. And no wonder, considering that from the sixteenth century onwards, religious, governance, and ultimately civic groups slowly accreted strategies for information verification and stabilisation (e.g., the imprimatur). But as with the previously mentioned issue of subject position and the algorithmic construction of audiences, texts, and stories, these inherited strategies are struggling to keep up with a new situation in which the provenance of online information is uncertain and correlations to events in the world are anyone's guess. Sure, fewer channels and centreto-periphery structures are easier to assess, characterise, and of course control than a networked free-for-all. But as we move ahead into this uncharted terrain, uncertainty seems all the more certain if we factor in the efforts of malevolent social actors and the steady advances of DeepFake technologies.

This condition of uncertainty takes three basic forms: uncertainty regarding what is real (an utterance's epistemological status in the age of digital manipulation); uncertainty regarding how users might assess this status (the source and 
logics behind the appearance of a particular utterance in a particular context); and uncertainty regarding implication (does the system "learn" from a user's pattern of likes, rejections, forwards, and contacts? And if so, with whom and to what end is that information shared, and how might it feed back into the flow of utterances that constitute the user's world? And does anyone else know what the user knows in an increasingly personalised world?). The good old days of Stuart Hall's dominant, negotiated, and oppositional reading positions, from this perspective, have a nostalgic glow.

The epistemological status of the emergent media system, the audiences they create, and the texts and stories that they trade in, is up for grabs. And this is the reason I have privileged a discussion of this recursive condition, rather than addressing crucially important issues having to do with the growing pressures of multicultural audiences, globalisation and post-coloniality, environmental concerns, and more. To the extent that media are part of the equation, these basic epistemic issues impinge upon the representation of all these problems.

\section{Legacy}

What now? As media and communication studies approach late-middle age, ${ }^{4}$ is it time to put their accumulated knowledge and expertise out to pasture? What can be usefully brokered from work in legacy media ecosystems that will be of use in the future? As already noted, the situatedness and therefore limits of inherited language and conceptual frames weigh heavily on the discussion. Wittgenstein's (1922: 162) injunction "Wovon man nicht sprechen kann, darüber muss man schweigen" [What we cannot speak about, we must pass over in silence] notwithstanding, the field will benefit from exploring the limits of the sayable and working towards the development of new critical vocabularies. These developments infuse even the most stubbornly entrenched media, shaping the realities of their production and distribution and defining their context. A few thoughts follow.

The close and critical reading of texts has a well-established tradition in film and television studies, and it's been interesting to observe the challenges faced by game scholars working with the variable textual utterances that emerge from the interaction of rule sets, assets, and player actions. The larger field needs to be attentive to what's working and what's not, as well as to which strategies might carry over to the domain of code and what I have referred to as the algorithmic systems in today's media. These elements and their operations need a nuanced descriptive apparatus, and it will require a radically expanded literacy if they are to be apprehended (see Montfort, 2016).

Part of this expanded literacy will require a shift from the familiar terrain of representation (where much work remains to be done, particularly in parsing modes like parody, satire, and "fakes") to include the domain of recognition, that is, to the systems and protocols that selectively capture, correlate, and "apprehend" the world. What, precisely, do they "see"? Who designs these systems, and 
with what remit? How are these systems trained? What are system limits, and how can the field develop a descriptive vocabulary to take this on? Joy Buolamwini's work on racial discrimination in facial recognition algorithms offers an inspiring example of what is possible. ${ }^{5}$

Reception research and a better understanding of how audiences are constructed, interpolated, and ultimately how they see themselves is essential as "personalised" media texts and delivery systems pervade the culture. The deeply qualitative audience research traditions associated with media studies offer an advantage with groups who are, as Sherry Turkle characterised them, "alone together". User-experience designers, who assess a broader range of activities than semiotic engagements alone, will also be particularly interesting to watch and learn from.

Studies of the political economy of the media are urgently needed as concentration continues to grow in the legacy sector (where cross-ownership of media forms as well as vertical integration of content producers and distribution channels has been accelerating since the 1980s). But these massive (and frankly worrisome) mergers are outpaced by the market value of Microsoft, Apple, Amazon, and Alphabet, which top the charts. Globalisation adds a curious twist, not only in the form of competitive entities such as China's Tencent and Alibaba, but in terms of control over content if producers seek access to large markets (Chinese box office revenues significantly outpaced American returns for the first time in 2018 and the trend has only been growing, as has the state's insistence on information control).

Given these concerns, at least some elements of continuity will be useful as the field develops new ways to address profound conditional changes. And it is here that the Nordic approach offers a way to transcend the divides (humanities and social science; media and communication studies; theoretical and empirical approaches) that characterise too much of the field. Of course, a journal devoted to Nordic media studies will rightly address the concerns of a region, reflect a cultural perspective, and even embody an ethos. But the demands of the new situation I've sketched - the paradigm shift of an algorithmically-enabled recursive regime evident in social media and emerging narrative operations - can benefit significantly from the generative and more wholistic approach that distinguishes the Nordic from many of its Anglo-American and European peers. I see the Nordic Journal of Media Studies as a forum for exchange that draws on this distinctive intellectual tradition and that will offer a crucial vantage point as the field develops strategies to broker past and present into a future capable of critical response.

\section{Notes}

1. I usually hew to Raymond Williams' notion of a medium as a technology and cultural form, or Lisa Gitelman's formulation, as a technological platform and social protocol expressed in a particular cultural-historical configuration, but for the purpose of this argument I take a smaller view. Media obviously precede the modern in the sense that I am using it, and certainly have a robust history outside the West, the domain of the "commonplace" to which I am referring.

2. For a curated compendium of examples, see the MIT Open Documentary Lab (http://docubase. mit.edu). 
3. For example, Karen Palmer's RIOT (2017+, found at http://karenpalmer.uk/portfolio/riot/), essentially a branched video narrative, uses a facial emotion recognition system to calculate the user's responses to the unfolding imagery, routing the user through the narrative environment accordingly. One could imagine this responsive layer being used in tandem with a predictive data set acquired from past behaviours, combining to construct an experience for the algorithmicallyextrapolated audience member.

4. At least from an American perspective, Film Studies penetrated the university in a systematic way in the 1960s, and the shift from the study of a medium to media studies (television) occurred in the late 1970s. Communication studies are significantly older, with roots at the turn of the century and institution-building in the 1920s and 1930s.

5. See in particular her Algorithmic Justice League (https://www.ajlunited.org/).

\section{References}

Cizek, K., \& Uricchio, W. (2019). Collective wisdom: Co-creating media within communities, across disciplines, and with algorithms. MIT Press PubPub. https://wip.mitpress.mit.edu/collectivewisdom

Gillespie, T. (2016). Algorithm. In B. Peters (Ed.), Digital keywords: A vocabulary of information society and culture (pp. 18-30). Princeton: Princeton University Press.

Johns, A. (1998). The nature of the book: Print and knowledge in the making. Chicago: University of Chicago Press.

Montfort, N. (2016). Exploratory programming for the arts and humanities. Cambridge, Massachusetts: MIT Press.

Uricchio, W. (2011). The algorithmic turn: Photosynth, augmented reality and the state of the image. Visual Studies, 26(1), 25-35. https://doi.org/10.1080/1472586X.2011.548486

Uricchio, W. (2017). Data, culture and the ambivalence of algorithms. In M.T. Schäfer, \& K. van Es (Eds.), The datafied society: Studying culture through data (pp. 125-138). Amsterdam: University of Amsterdam Press. http://library.oapen.org/handle/20.500.12657/31843

Wittgenstein, L. (1922). Tractatus logico-philosophicus. London: Kegan Paul, Trench, Trubner \& Co.

Copyright: ( 92020 The Author(s) and Nordicom. This is an Open Access article distributed under the terms of the Creative Commons Attribution 4.0 International License (CC BY-NC-ND 4.0). 